\title{
Venusinae Musae amatoribus
}

Württembergische Neulateiner zu Anfang des 19. Jahrhunderts

\author{
Von Walther Ludwig
}

Gustav Schwab (1792-1850) ${ }^{1}$, seit 1818 ein junger Professor für Latein und Griechisch am Oberen Gymnasium in Stuttgart ${ }^{2}$, heute vor allem bekannt durch den auflagenreichen Dauerbrenner „Die schönsten Sagen des klassischen Alterthums“, die es seit 2009 sogar als Hörbuch und seit 2011 auch in einer Auswahlausgabe gibt, veröffentlichte 1823 in Stuttgart bei Cotta 38 Seiten in Quarto $(22,5 \times 17 \mathrm{~cm})$ mit folgendem Titel $^{3}$ :

LVDOVICI VHLANDI|| DE || CONSTITVENDA RE PVBLICA CARMINA.| LATINITATE ET METRIS HORATIANIS VESTITA || VENVSINAE MVSAE AMATORIBVS OFFERT\| ADIECTO TEXTV VERNACVLO\| GVSTAVVS SCHWAB.|| STVTGARDIAE || IN LibRARIA COTTAE.|| MDCCCXXIII.

Die deutsche Vorlage für diese breitrandig gedruckte lateinische Versübersetzung waren die kleiner unter dem lateinischen Text jeweils abgedruckten „Vaterländische(n) Gedichte“ Ludwig Uhlands (1787-1862), die in der Zeit der württem-

1 Vgl. Nikolaus GatTer, Art. „Schwab, Gustav“, in: NDB, Bd.23, Berlin 2007, S. 772773. - Hermann Fischer, Art. „Schwab, Gustav“, in: ADB, Bd. 33, Leipzig 1891, S. 153-155. - Karl Klüpfel, Gustav Schwab. Sein Leben und Wirken, Leipzig 1858.

2 Das Gymnasium wurde nach Gründung des dortigen Karls-Gymnasiums 1881 Eberhard-Ludwigs-Gymnasium genannt. In allen Klassen des Gymnasiums wurden im 19. Jahrhundert von montags bis samstags jährlich ca. 12-8 Wochenstunden Latein und von der Mittelstufe an ca. 6 Wochenstunden Griechisch und ca. 3 Wochenstunden Französisch sowie in der Oberstufe ca. 3 Wochenstunden Hebräisch und ca. 2 Wochenstunden Englisch unterrichtet; vgl. die Programme des Königlichen Gymnasiums in Stuttgart seit 1863 und die Programme des Eberhard-Ludwigs-Gymnasiums in Stuttgart seit 1881 sowie Gustav LANG, Geschichte der Stuttgarter Gelehrtenschule von ihren ersten Anfängen bis zum Jahre 1806, in: Geschichte des humanistischen Schulwesens in Württemberg, hg. von der Württembergischen Kommission für Landesgeschichte, Bd.3, 2, Teil 1, Stuttgart 1928 (Teil 2 nicht erschienen).

3 Bernhard Fischer, Der Verleger Johann Friedrich Cotta - chronologische Verlagsbibliographie 1787-1832, Bd. 2: 1815-1832, Stuttgart 2003, S. 372, Nr. 1453. 
bergischen Verfassungskämpfe seit 1815 entstanden waren (mit „Vaterland“ ist hier wie damals üblich Württemberg gemeint). Schwab benützte aber nicht die Erstausgabe, die 1817 erschienen war und die elf 1815-1817 komponierte Gedichte enthält, sondern die Ausgabe „Gedichte von Ludwig Uhland. Zweite vermehrte Auflage, Tübingen 1820“, in die diese „Vaterländische(n) Gedichte“ in einer durch drei 18171819 verfasste Gedichte vermehrten Form aufgenommen worden waren ${ }^{4}$, so dass sein Druck 14 Gedichte umfasst.

Mit dem lateinischen Titel wandte sich Schwab nicht nur an die Liebhaber der Horazischen Gedichte ${ }^{5}$, sondern erklärte sich implizit auch selbst zu einem Venusinae Musae amator 6 . Am 11. April 2014 gelangte bei Bassenge, Berlin, ein Exemplar dieses Drucks zur Versteigerung ${ }^{7}$, das auch eine handschriftliche Widmung von Schwab enthält. Sie steht auf der Innenseite des vorderen Umschlagpapiers des gehefteten Drucks und lautet: „Sr. Hochwürden Herrn Prof. Jäger| hochachtungsvollst zugesandt| vom Übersetzer." Der Empfänger war Dr. med. Georg Friedrich Jäger (1785-1866), der, promoviert in Tübingen 1808, 1817-1856 Leiter des Naturalienkabinett genannten Stuttgarter Naturkundemuseums und 1822-1846 Professor der Chemie und Naturgeschichte am Oberen Gymnasium in Stuttgart war und 1841 dort auch Obermedizinalrat wurde. Er war also 1823 Schwabs Kollege am Oberen Gymnasium und, da er 1819 in zweiter Ehe Charlotte Wilhelmine Schwab (1794-1874), eine Schwester von Gustav Schwab, geheiratet hatte, zugleich dessen

${ }^{4}$ Ludwig Uhland, Gedichte. Zweite vermehrte Auflage, Tübingen 1820, S. 97-121 und 463-464. Fischer, Der Verleger Johann Friedrich Cotta (wie Anm. 3) nennt die Erstauflage als eine Vorlage. In der „Neueste(n) Auflage“ der Gedichte von Ludwig Uhland, Stuttgart/ Tübingen 1839, S.137, findet sich erstmals ein 15. Gedicht bei den „Vaterländische(n) Gedichten", womit diese Sammlung ihren endgültigen Umfang erreicht hatte. So stellt sie sich auch in der „Vierundfünfzigste(n) Auflage“ der Gedichte von Ludwig Uhland, hg. von Wilhelm Ludwig Holland, Stuttgart 1869, S. 81-106 dar, von der ein Freund von Uhland, der Rottweiler Rechtskonsulent Dr. iur. Karl Friedrich Rheinwald (1802-1876), der 1848/1849 auch Mitglied der Nationalversammlung in Frankfurt und Stuttgart auf Seiten der Demokraten gewesen war, ein Exemplar seinem vierzehnjährigen Neffen und Patensohn Karl Friedrich Rudolf Ludwig (1855-1929) zu Weihnachten 1869 schenkte. Vgl. zu Rheinwald und Ludwig: Walther Ludw IG, Rudolf Lohbauers Bild ,Hyperions Fahrt nach Kalaurea', in: Hölderlin-Jahrbuch 30 (1996-1997) S. 359-380, hier S. 373-377. Dieses Exemplar befindet sich im Besitz des Verfassers.

5 Musa Venusina (basierend auf Juvenal Sat. 1, 51 Venusina digna lucerna) war in der neulateinischen Literatur eine beliebte Umschreibung für die horazische Dichtung geworden. Fast in jeder Horazausgabe las der Leser vor den Gedichten eine mit den Worten Q. Horatius Flaccus Venusinus beginnende oder den Geburtsort Venusia am Anfang direkt nennende Vita.

${ }^{6}$ Schwab hätte deshalb mit gutem Recht bereits in meinem Aufsatz „Die Liebe zu Horaz - Horaz in der Kultur der europäischen Neuzeit" genannt werden können, s. Walther LudW IG, Opuscula historico-philologica. Ausgewählte Aufsätze 2008-2013, ebd. hg. von Astrid Steiner-Weber, Hildesheim u. a. 2014 (Noctes Neolatinae 19), S. 71-102.

7 Bassenge Buchauktionen GbR, Auktion 103, Berlin 2014, Nr. 2334. Das Exemplar befindet sich im Besitz des Verfassers.

Zeitschrift für Württembergische Landesgeschichte 74 (2015), S. 121-148.

(C) Kommission für geschichtliche Landeskunde in Baden-Württemberg und Württembergischer Geschichts- und Altertumsverein e.V.

ISSN 0044-3786 
Schwager ${ }^{8}$. Den heutigen Leser wird bei diesen Beziehungen zwischen den beiden die offenbar der Zeit gemäße Förmlichkeit von Schwabs Widmung überraschen. Jägers Sohn, der Philologe und Kölner Gymnasialdirektor Oskar Jäger (18301910), wird 1905 über seines Vaters Verhältnis zu Horaz schreiben: „In alten Tagen geschah es [sc. das Auswendiglernen einzelner Oden] freiwillig, ohne Befehl und Mahnung wie jetzt im preußischen Lehrplan S. 27 „Auswendiglernen einzelner seiner Oden“: ich bewahre noch das schlicht gebundene, schlechtgedruckte Bändchen [sc. der Horazischen Gedichte], das meinen Vater, einen Arzt und Naturforscher, 50 Jahre lang auf seinen Reisen begleitete" ${ }^{\text {" }}$. Georg Friedrich Jäger hatte diese Horazausgabe also schon etwa 1816 erworben (vielleicht bereits früher, wenn sie ihn auf seinen Reisen begleitete -1809 reiste er auf seiner größten Reise nach Paris, durch Südfrankreich und die Schweiz), und er besaß sie sicher schon 1823, als er von Schwab dessen horazisierende Versübersetzung der Gedichte von Uhland erhielt, mit dem Jäger seinerseits seit seiner Studentenzeit befreundet war. Schwab konnte davon ausgehen, daß Jäger, der wie Schwab als Schüler auch das Stuttgarter Gymnasium besucht hatte, selbst zu den Venusinae Musae amatores gehörte.

Uhland begann seine „Vaterländische(n) Gedichte“ im Oktober 1815 mit einem offenen Gedicht an den Stuttgarter Bürgermeister Heinrich Immanuel Klüpfel $(1758-1823)^{10}$, der zum Vertreter Stuttgarts in der württembergischen Ständeversammlung gewählt worden war und für den am 18. Oktober ein Fest gegeben wur$\mathrm{de}^{11}$. König Friedrich I. von Württemberg (1754-30.10.1816) hatte im Dezember 1805 die auf dem Tübinger Vertrag von 1514 beruhende württembergische Ständeverfassung durch einen Rechtsbruch aufgehoben, und eine neu einberufene württembergische Ständeversammlung, zu der Klüpfel gehörte, hatte im März 1815 die ihr nun vom selben König zum zustimmenden Schwur vorgelegte neue württembergische Verfassung einhellig abgelehnt. Die jetzt ausbrechenden und erst unter König Wilhelm I. (1781-1864) am 25. September 1819 durch den Kompromiss einer neuen Verfassung beigelegten Verfassungskämpfe wurden begleitet und unterstützt von einzelnen Gedichten Uhlands, in denen er sich für „das alte gute Recht“ einsetzte und von denen er weiter neun im Jahr 1816 und eines im März

8 S. Wilhelm von Gümbel, Art. „Jaeger, Georg Friedrich von“, in: ADB, Bd. 13, Leipzig 1881, S. 648-649. - Karl Staesche, Art. „Jäger, Georg Friedrich von“, in: NDB, Bd. 10, Berlin 1974, S. 268-269. Vgl. auch Gustav Sch wa B, Zur Feier der Hochzeit des Herrn Georg Jäger, der Medizin Doctor, mit Jungfrau Charlotte Schwab, Stuttgart 1819, [8] S., vorhanden: Stuttgart, Landeskirchliche Zentralbibliothek, Signatur: AS/11111.

9 Oskar JäGER, Homer und Horaz im Gymnasial-Unterricht, München 1905, S. 179, und Ludwig, Opuscula (wie Anm. 6) S. 94. Wahrscheinlich erbte Oskar Jäger auch diesen Druck der lateinischen Gedichte von Schwab.

10 Vgl. Karl KLÜPFEL, Art. „Klüpfel, Heinrich Immanuel“, in: ADB, Bd. 16, Leipzig 1882, S. 257-258.

${ }^{11}$ Vgl. Emilie Uhland, Ludwig Uhlands Leben, Aus dem Nachlaß und aus eigener Erinnerung zusammengestellt von seiner Wittwe, Stuttgart 1874, S. 113, und dort zu den übrigen Vaterländischen Gedichten S. 113-165. 
1817 verfasste und teilweise einzeln publizierte, um die elf Gedichte dann zusammen unter dem Titel „Vaterländische Gedichte“ auch geschlossen zu veröffentlichen. Es folgten 1817 von April bis Juni noch zwei Gedichte mit dem gleichen Ziel und schließlich am 29. Oktober 1819 ein vorerst letztes als Prolog zu Uhlands damals im königlichen Hoftheater in Stuttgart aufgeführtem Trauerspiel „Ernst, Herzog von Schwaben“"12. In diesem Theater-Prolog wird nun der Festtag der neuen Verfassung gefeiert, und er endet versöhnlich mit den Worten „Heil diesem König, diesem Volke Heil! “13. Diese politischen Gedichte sind in verschiedenen Stilebenen, zu einem großen Teil in einem volkstümlichen Ton gehalten und bis auf das letzte, das in Blankversen vorgetragen wurde, in männlichen und weiblichen Versen mit drei bis vier Hebungen und in Strophen, die meist nach dem Schema abab oder ababcc, einmal nach abba-Reimen, verfasst.

Seine Verehrung für und Freundschaft mit Uhland ${ }^{14}$, die Verfassungsfrage als das 1815-1819 wichtigste politische Thema im Königreich Württemberg und seine Liebe zu Horaz ${ }^{15}$ trugen dazu bei, dass Schwab sich entschloss, die „Vaterlän-

12 Vgl. Ludwig Uhland, Ernst, Herzog von Schwaben. Trauerspiel in fünf Aufzügen, Heidelberg 1818. Der genannte Prolog ist in dieser Ausgabe jedoch nicht enthalten. Er wurde zuerst gedruckt in UHLAND, Gedichte (wie Anm. 4) S. 463-464.

13 Vgl. Emilie Uhland, Ludwig Uhlands Leben (wie Anm.11) S. 165: „War auch nicht alles erreicht, was Uhland gew ünscht hatte, war auch die Adelskammer gegen seinen Sinn, so war doch die Verfassung auf das alte Recht, durch Vertrag zwischen Fürst und Volk neu begründet. Darauf legte Uhland, wie wir wissen, wie er in seinem Liede ausgesprochen, so großes Gewicht.“ und allgemein Günter Grossвach, „... und aufrecht, wie ihn Gott erschuf." Uhland in den württembergischen Verfassungskämpfen 1815-1819 und als württembergischer Abgeordneter, in: Ludwig Uhland. Werk und Wirkung. Festschrift des Uhland-Gymnasiums Tübingen zum 200. Geburtstag des Politikers, Gelehrten, Dichters, hg. von Uhland-Gymnasium Tübingen, Redaktion: Dr. Helmut Sтовсн, Tübingen 1987, S. 1939, sowie für eine Abbildung des „Verfassungs-Werks für das Königreich Württemberg“: Das Königreich Württemberg. 1806-1918. Monarchie und Moderne, hg. vom Landesmuseum Württemberg, Ostfildern 2006, S. 92-94. Die Entstehungsdaten der einzelnen Gedichte Uhlands werden von Holland (wie Anm. 4) S. 478-479, angegeben.

${ }^{14}$ Klüpfel, Gustav Schwab (wie Anm.1) S. 49: „seine Beziehung zu Uhland ... entwickelte sich mehr und mehr zu einer innigen Freundschaft." S. 30 zufolge war Schwab Uhland zuerst 1811 in Tübingen begegnet.

15 Welche Ausgabe bzw. welche Ausgaben des Horaz Schwab benützte, ist unbekannt. Die zahlreichen Klassikerausgaben der in Zweibrücken tätigen Societas Bipontina, darunter die Editio accurata der Opera des Horaz von 1783, waren damals auch in Württemberg verbreitet. So besaß z.B. der Tübinger Hofgerichtsadvokat und Lizentiat der Rechte Eberhard Friedrich Hehl (1765-1847), der als Abgeordneter in der württembergischen Ständeversammlung für die Stadt Tübingen am 25. September 1819 das neue „Verfassungs-Werk für das Königreich Württemberg" mitunterzeichnete, eine umfangreiche Sammlung dieser Ausgaben, darunter auch die genannte Horazausgabe (auf dem Vorsatz gezeichnet mit „Hehl d. R.L. 22. Nov. 1783“). Seine Witwe gab 1847-1849 zahlreiche Bücher und Manuskripte aus seinem Besitz an die Universitätsbibliothek Tübingen (Handbuch der historischen Buchbestände. Baden-Württemberg und Saarland. T-Z, hg. von Wolfgang KeHr, Hildesheim u.a. 1994, S. 27), seine Bipontinen-Sammlung wurde jedoch privat vererbt und befindet sich jetzt teilweise im Besitz des Verfassers. 
dische(n) Gedichte“ Uhlands in horazische Verse umzusetzen. Auch Horaz hatte bekanntlich - nicht nur in den sogenannten Römeroden - ernste politische Gedichte geschrieben. Um das Thema der Uhlandschen Gedichte und ihre Bedeutung sogleich deutlich zu machen, nannte Schwab sie nicht mit einfacher Übersetzung des Uhlandschen Titels Carmina patria, sondern Carmina de constituenda re publica. Er konnte für den Druck seines Werkes den angesehenen Cotta-Verlag in Stuttgart gewinnen und erhielt bald nach dem Erscheinen der Carmina zwei ausführliche positive (namentlich nicht gezeichnete) Besprechungen in der Allgemeinen Literaturzeitung Halle 1823, II, Sp. 865-868, und in den Heidelberger Jahrbüchern der Literatur N. F. 4 I, Heidelberg 1824, S. 350-352, aus denen einige Zeilen hier zitiert seien:

„Man hat seit einiger Zeit wieder angefangen, wie früher schon von Spalding, dem Übersetzer des Kleistischen Frühlings u. a. geschehen, die lateinische Dichtkunst im Wettstreit mit der deutschen auftreten zu lassen. An Gedichten von Klopstock, Schiller, ja an größeren von Voß und Göthe haben sich vor kurzem mehrere römische Verskünstler versucht. Solche Uebungen sind immer zu empfehlen, und dienen nicht nur zur Belebung des Studiums alter Sprache und Poesie, sondern sie verhelfen auch, wenn sie von gewandten, berufenen Männern unternommen sind, dem philosophischen Sprachforscher selbst zu gelegenheitlicher näherer Prüfung des Geistes, des Baues und der Eigenthümlichkeiten verschiedner Sprachen. Hr. Professor Schw. als talentvoller, frischer deutscher Dichter ebenso als einsichtsvoller Humanist durch deutsche und lateinische Schriften bekannt, hat sich keine leichte Aufgabe gegeben, die vaterländischen Gedichte seines Freundes Uhland, die zuerst in einer besonderen Ausgabe (Tübingen, bei Fues) und dann in der neuen vermehrten Sammlung der Uhland'schen Gedichte erschienen sind, in Horazischen Sylbenmaassen nachzubilden. Nachzubilden sagen wir: denn eigentliche Übersetzungen im strengeren Sinn sind diese lateinischen Gedichte nicht und sollten es auch nicht seyn. Da die Uhland'schen schon früher von uns ihrem gediegenen Gehalt gemäss in diesen Blättern empfohlenen Gedichte alle in gereimten zum Theil volksthümlichen Sylbenmaassen componirt sind, so wäre es eine Art Prokrusteischer Arbeit gewesen, wenn Hr. Schw., etwa, wie bey der Schiller'schen Glocke von Niethammer nicht ohne Glück, dorten doch wegen der abwechselnden Versart an einem leichteren Vorbilde versucht worden ist, die kurzen Jambischen u.a. Versmaasse seines Urbildes hätte in ähnlicher gereimter lateinischer Poesie nach Weise der Mönchspoesie wiedergeben wollen. Auch der Inhalt selbst hätte sich nicht recht dafür geeignet, was bey der Schiller'schen Glocke schon ein anderer Fall ist, die ursprünglich sozusagen in einen mittelalterlichen Ton eingestimmt ist. Es war daher von Hn. Schw. ein glücklicher Gedanke, dass er das hierher Ungehörige und an sich Unmögliche einer strengeren Uebersetzung nach Form und Inhalt verschmähend, bey den antiken Sylbenmaassen um Surrogate sich umsah, nicht ohne jemalige einsichtsvolle Berechnung, welche derselben für die Erfassung und analoge Darstellung des Tons der Originale am geeignetsten wären, und so eher zu 
einer freyeren Nachbildung sich entschloss. Jedes Gedicht ist beinahe in einem anderen Sylbenmaasse übersetzt worden. [...]“

„Wir dürfen wohl voraussetzen, dass auch ausserhalb Württemberg die im Jahre 1817 in Tübingen bei Fues herausgekommenen ,Vaterländischen Gedichte ${ }^{6}$ von Ludwig Uhland sich verbreitet haben, und mit verdienter Theilnahme gelesen worden seyen. Von diesen nun sind die vorliegenden Carmina de constituenda re publica eine sehr gelungene Uebersetzung, die wir dem Freunde L. Uhlands, Hrn. Prof. Schwab in Stuttgart verdanken. [...] Der Uebersetzer, mit des Dichters Sinn und Geist und Darstellung vertraut und verwandt, beweisst zugleich eine ausgezeichnete Bekanntschaft mit der Horazischen Muse, und die Leichtigkeit, Natur und Anmuth, die uns in seinen eigenen Gedichten anzieht, finden wir auch in dieser Uebersetzung, bei der nicht wenige und nicht geringe Schwierigkeiten zu überwinden waren, wenn sie so römisch und doch so treu ausfallen sollte, wie sie vor uns liegt. Gelungen nennen wir diese Uebertragung mit Recht darum, weil sich in ihr durchaus das findet, was gefordert werden muss, nemlich auf der einen Seite ein Vergessen deutscher Art und Kunst, und auf der andern doch zugleich ein Festhalten an den Ideen, ja sogar, wo es möglich war, an den Worten, so daß fast Zeile für Zeile dem Original ähnlich ist. [...] Ausser dem Wert, den die Uebersetzung an sich hat, kommt ihr zugleich der zu, dass sie jungen Studirenden als Muster dienen kann, wenn sie sich in Horazischen Versmassen versuchen wollen.“

Für diese Besprechungen ist das Vorhaben einer lateinischen Dichtung in horazischen Versmaßen nicht ungewöhnlich. Andere lateinische Versübersetzungen von deutschen Dichtungen werden zum Vergleich herangezogen. Auf letztere wird im Folgenden noch einzugehen sein. Später fanden die Versübersetzungen Schwabs nur noch wenig Beachtung ${ }^{16}$. Schon Rudolf Krauß (1861-1945) erwähnte sie in seiner berühmten „Schwäbische(n) Literaturgeschichte“ 1899 nur noch in einem Satzteil: „zeigte sein philologisches Können in Abhandlungen, hauptsächlich über Sophokles, und einer Latinisierung der politischen Gedichte Uhlands s'17. Und Gunter Grimm wußte 1992 in einem Artikel „Zum zweihundertsten Geburtstag des Schriftstellers und Volkserziehers Gustav Schwab “18 sogar nur noch „Anthologien griechischer, lateinischer und deutscher Gedichte“ zu nennen, wobei sich hinter

16 Aufgenommen wurde Schwabs Werk in die Liste der lateinischen Versübersetzungen deutscher Dichtungen von Theodorus Echtermayer/Mauritius Seyffert, Carmina aliquot Goethii et Schilleri latine reddita, Halle 1833, S. IV-V, und in die nützlichen bibliographischen und biographischen Zusammenstellungen, die Bernd Platzdasch unter dem Titel „Pantoia, Unterhaltsame Literatur und Dichtung in lateinischer und griechischer Übersetzung“ ins Internet gestellt hat (Fassung vom 22.2.2012: www.pantoia.de/pantoia.html).

17 Rudolf Krauss, Schwäbische Literaturgeschichte, 2. Bd., Freiburg i. Br./Leipzig/Tübingen 1899, S. 69.

${ }_{18}$ Gunter Grimm, Das Faktotum der Musen. Zum zweihundertsten Geburtstag des Schriftstellers und Volkserziehers Gustav Schwab, in: Die Brücke zur Welt, Sonntagsbeilage der Stuttgarter Zeitung, Samstag, 20. Juni 1992 (im Internet: http://duepublico.uni-duisburg-essen.de/servlets/DerivateServlet/Derivate-5357/Schwab.pdf). 
der vermeintlichen und an sich nicht existenten Anthologie lateinischer Gedichte wohl Schwabs Versübersetzung der Gedichte Uhlands verbirgt. Allein Helmut Storch machte 1987 kurz auf diese Versübersetzung aufmerksam und zitierte, allerdings ohne weitere Erläuterungen, die vier Strophen des 10. Gedichts mit dem Uhlandschen und dem Schwabschen Text ${ }^{19}$. Die Schwabsche Versübersetzung soll jedoch hier im Rahmen einer Musterung des neulateinischen Dichtens in Württemberg in den ersten Jahrzehnten des 19. Jahrhunderts zunächst etwas näher betrachtet werden.

Schwab verzichtete auf jede begründende und rechtfertigende persönliche Praefatio. Er wusste vermutlich, dass Uhland schon selbst lateinische Oden in horazischer Form verfasst hatte ${ }^{20}$, und wünschte, dass die 14 Gedichte eine repräsentative Auswahl der Oden- und Epoden-Metren des Horaz enthalten sollten. Die beiden häufigsten horazischen Metren, die alkäische und die sapphische Strophe, hob er hervor, indem er nur für diese Strophen mehrere Gedichte, insgesamt fünf, verwendete und indem er die alkäische Strophe an den Anfang und das Ende der um das alte gute Recht kämpfenden Gedichte setzte (Nr. 1 und 13). Dazwischen kamen drei sapphische Gedichte zu stehen (Nr. 3, 5 und 12). Für das 14. Gedicht, den Prolog zur Aufführung des Trauerspiels Herzog Ernst am 29. Oktober 1819, wählte er passend das Metrum für den Sprechvers der Tragödien Senecas, den jambischen Trimeter, der aber zugleich auch das Metrum der letzten Epode des Horaz (Epd. 17) war. Die fünf verschiedenen asklepiadeischen Systeme des Horaz sind jeweils durch ein Gedicht vertreten: das Asclepiadeum quartum (Glyconeus + Asclepiadeus minor) in Nr. 4 (hier spielte wohl auch eine Rolle, dass das Gedicht ein Dialog ist wie C. III 9, das die gleiche Strophenform hat), das Asclepiadeum secundum (3 Aslepiadei minores + Glyconeus) in Nr. 6, das Asclepiadeum primum (Asclepiadei minores stichisch) in Nr. 8, das Asclepiadeum quintum (Asclepiadei maiores stichisch) in Nr. 9 und das Asclepiadeum tertium (Asclepiadeus minor + Pherecrateus + Glyconeus) in Nr. 10. Zu diesen zehn Odenformen kommen noch insgesamt vier horazische Epodenformen, von denen eine, bestehend aus einem daktylischen Hexameter und einem katalektischen daktylischen Tetrameter, bei Horaz nicht nur in Epode 12 erscheint, sondern strophisch auch in zwei Oden (C. I 7 und 28; dort mit der metrischen Bezeichnung Archilochium primum). Schwab hat dieses die Oden und Epoden verbindende Metrum für Nr.7 gewählt, auffälligerweise für das Gedicht im Zentrum von Nr.1-13. Des weiteren erscheint die aus den Epoden 14 und 15 bekannte Kombination von daktylischem Hexameter und jambischem Dimeter gleich in Nr.2, die bei Horaz in den Epoden 1-10 verwendete Kombination eines jambischen Trimeters und eines jambischen Dimeters in Nr.11, und, wie schon notiert, der stichische jambische Trimeter von Epode 17 in Nr. 14. Die folgende

19 Helmut Storch, Gustav Schwab, Zwei Gedichte, das eine von Uhland, ins Lateinische gewendet - das andere auf Uhland, in: Ludwig Uhland (wie Anm. 13) S. 40-44.

${ }^{20}$ Hermann Steinthal, Die Tübinger schola anatolica um 1800 und ihr Schüler Ludwig Uhland, in: Ludwig Uhland (wie Anm. 13) S. 1-18, hier S. 4. 
Übersicht über die Metren der 14 Gedichte kann vielleicht noch deutlicher machen, wie Schwab in seinen Carmina die häufigeren horazischen Oden- und Epodenmaße vereinen und in eine gewisse Ordnung bringen wollte:

1. Alcaicum

56 Verse

3. Sapphicum

2. Dact. hex. + ia. dim. (Epd. 14 und 15)

44

40

4. Asclepiadeum quartum

28

5. Sapphicum

20

6. Asclepiadeum secundum

56

7. Archilochium primum $=$ Epd. 12

(Dact. hex. + dact. tetram. cat.)

16

8. Asclepiadeum primum

8

9. Asclepiadeum quintum

18

10. Asclepiadeum tertium

16

11. Ia. trim. + ia. dim. (Epd. 1-10) 32

12. Sapphicum

13. Alcaicum

14. Ia. trim. (Epd. 17)

Um nun einen ersten Eindruck von der Art dieser horazischen Gedichte zu gewinnen, betrachten wir die ersten vier der 14 alkäischen Strophen des ersten Gedichts, die zwei achtzeiligen Strophen Uhlands entsprechen:

Die Schlacht der Völker ward geschlagen,

Der Fremde wich von deutscher Flur,

Doch die befreiten Lande tragen

Noch manches vorgen Dranges Spur,

Und wie man aus versunknen Städten

Erhabne Götterbilder gräbt,

So ist manch heilig Recht zu retten,

Das unter wüsten Trümmern lebt.

$\mathrm{Zu}$ retten gilts und aufzubauen;

Doch das Gedeihen bleibet fern,

Wo Liebe fehlet und Vertrauen

Und Eintracht zwischen Volk und Herrn.

Der Deutsche ehrt' in allen Zeiten

Der Fürsten heiligen Beruf,

Doch liebt er, frei einherzuschreiten

Und aufrecht, wie ihn Gott erschuf. 
Conjunctus orbis proelia miscuit,

Germana tellus propulit exteros,

Sed mansit in terra soluta

Servitii nota multa prisci.

Atque ut Deorum signa per obrutas

Urbis ruinas effodiunt sacra:

Coeleste jus passim sepultum

Ruderibus viget indecoris.

Servate, cives! tollite moenia!

Sed, heu, sinistro conditis alite,

Ni principis, ni corda gentis

Jungit amor, fideique vinclum.

Sacrata Teuto munia principum

Veretur omni tempore, idem ${ }^{21}$ tamen

Erecta - sic natura jussit -

Ferre amat ora, gradique liber.

„Der vereinte Erdkreis stürzte sich in die Schlacht“. Gemeint ist die Völkerschlacht von Leipzig, die am 18. Oktober 1815, dem Tag des Gedichts, genau zwei Jahre zurücklag. Der Ausdruck proelia miscuit schließt sich an den von Lukrez und Vergil gebrauchten Ausdruck proelia miscent an. Unmittelbar an Horaz erinnern in diesen Strophen ausdrucksmäßig nur die eingeschobene Klageinterjektion beu und der Ablativ alite im Sinne eines Vorzeichens, ohne dass hier bestimmte Stellen evoziert werden sollen. Auffällig ist die Häufigkeit der normalen Wortstellung. Die erste - kleine - Sperrung erscheint im 4. Vers, wodurch sich die kunstvolle Wortfolge nach dem Schema ABba einstellt. Eine große Sperrung zeigen V. 5-6 signa ... sacra (hier wird die kleine obrutas ... ruinas eingeschlossen) und V. 12-13 erecta ... ora. Jede Strophe ist syntaktisch in sich geschlossen und meist stellen auch die Verse oder doch die Verspaare syntaktische Einheiten dar. Ein starker syntaktischer Neueinsatz innerhalb eines Verses liegt nur bei idem tamen bzw. at is tamen vor. Schwab schließt sich hier nicht so sehr an den Stil des Horaz als an den von Uhland an.

${ }^{21}$ In dem in Anm.7 genannten Exemplar strich Schwab handschriftlich idem durch und schrieb at is darüber. Es ist die einzige Textkorrektur in diesem Exemplar. at is tamen bildet einen stärkeren Gegensatz als idem tamen. Vgl. Immanuel Johann Gerhad SchelLER, Lateinisch-deutsches Lexicon, Bd. 1, Leipzig 1788, Sp. 581: „Attamen, aber doch, allein doch, jedoch aber und richtiger at tamen, denn es sind zwei Wörter; daher stehen oft Wörter dazwischen, z. B. at pro nostro tamen studio Cic.Or.III 4: at grato tamen munere Cic. Brut. 4. "Für weitere Beispiele s. Thesaurus Linguae Latinae, Vol. 2, Leipzig 1900-1906, Sp. 1010-1011. 
Das das Grundthema explizierende zweite Gedicht („Das alte gute Recht“, De bono iure patrio) führt nach der strophischen sogleich die epodische Odenform ein. In seinen 44 Versen nimmt der Wortlaut auf zwei Horazstellen speziell Bezug. Die ersten vier Verse und die letzten vier (41-44) sind im Deutschen fast, im Lateinischen völlig identisch:

Wo je bei altem, gutem Wein (V. 41: Und wo bei altem, gutem Wein)

Der Württemberger zecht,

Da soll der erste Trinkspruch seyn:

Das alte, gute Recht.

Wurtembergiacae si quando quis incola terrae

Calescit antiquo mero:

Haec potatorum statuatur tessera princeps:

Antiqua jura patriae!

Zwischen diesen jeweils vier Versen werden mit vielen anaphorischen Versanfängen („Das Recht, ...“; Jura ,...) zuerst die Bestimmungen dieses Rechts beschrieben: gültige vor Willkür schützende Gesetze und öffentliche Gerichte, mäßige Steuern und ein sparsam wirtschaftender Staat, Bewahrung des Kirchenguts und Förderung der Wissenschaft, freier Waffenbesitz der Bürger [!] und ihre Auswanderungsfreiheit. Deshalb werde das alte gute Recht ebenso wie der christliche Glaube geliebt. In einer schlimmen Zeit (malum tempus - der Täter König Friedrich I. wird nicht erwähnt) sei es abgewürgt worden, und es erhebe sich jetzt wieder zu einem neuen Leben, das nach dem Tod der jetzigen Generation (V. 37: „Ja! wenn auch wir von hinnen sind“; Quod si nos tetigit rapiens vis omnia leti) andauern solle. V. 37 erinnert an Horaz C. II 13, 19-20 leti| vis rapuit rapietque gentes, V. 2 und 42 an C. III 21, 10-11 narratur et prisci Catonis| saepe mero caluisse virtus. Die Aufnahme bestimmter Stellen kommt also vor, ist aber vergleichsweise selten.

Es gelang Schwab, Uhlands Text prinzipiell mit ebenso vielen lateinischen Versen wiederzugeben ${ }^{22}$. In Nr. 7, 8 und 9 fasste er die 24, 16 bzw. 36 Kurzverse in 16, 8 bzw. 18 längeren Versen zusammen. In der Regel entsprechen einander Vers

22 Nur vereinzelt leidet unter diesem angenommenen Zwang, die Verszahlen des deutschen Gedichts einzuhalten, die Klarheit des Ausdrucks wie in der im Übrigen eindrucksvollen sapphischen Ode Nr.12 („Gebet eines Württembergers“, Ad Deum O.M.) in der zweiten Strophe (V. 5-8). „Zu unsrem König, deinem Knecht,| Kann nicht des Volkes Stimme kommen;| Hätt' er sie, wie er [sc. sie vernommen haben] will, vernommen,| Wir hätten längst das theure Recht." gibt Schwab mit Ad meum regem Tibi servientem| Ire vox frustra populi laborat, | Dulce jus (nam vult) simul audiisset,| Sanxerat ille wieder. V. 7-8 ist wohl so zu verstehen: Dulce jus sanxerat ille, simul ac vocem populi (nam vult andivisse) andiisset. „Das süße Recht hätte er gewiß bestätigt, sobald als er die Stimme des Volkes gehört hätte, die er ja gehört haben will." Der Indikativ des Plusquamperfekts ist in einem irrealen Bedingungssatz auch klassisch belegt. Aber die Wortstellung und der knappe Ausdruck verhin- 
für Vers im Sinn ziemlich genau. Der Anschluss an die Uhlandschen Verse verhinderte eine stärkere Aufnahme horazischer Motive und Strukturen, wie sie bei einem freien Komponieren horazisierender Oden eventuell möglich ist. Nur selten gibt Schwab sich bei besonderen Schwierigkeiten die Freiheit, ein Wort fallen zu lassen, so bei folgenden Versen in Nr. 6 („Am 18. October 1816“; In diem Octobris octavum decimum anni MDCCCXVI):

Ihr Fürstenräth' und Hofmarschälle,

Mit trübem Stern auf kalter Brust,

Die ihr vom Kampf um Leipzigs Wälle

Wohl gar bis heute nichts gewußt,

Regni a consiliis denique vos, quibus

Aurum corda tegit frigida pallidum,

Qui fortasse bonae proelia Lipsiae

Ignorastis ad hunc diem

Die erste Zeile hätte prosaisch mit Vos principum consiliarii et aulae praefecti wiedergegeben werden können. In eine einzige Verszeile passten die beiden Hofchargen keinesfalls. Schwab entschloss sich, auf die Hofmarschälle zu verzichten und zog für die Hofräte die nach der antiken Amtsbezeichnung ab epistulis im neuzeitlichen Latein schon eingeführte Bezeichnung a consiliis vor ${ }^{23}$. Er hatte nun Raum, um in der zweiten Zeile für die Ordensbrust die elegante neue Junktur und Wortstellung aurum corda tegit frigida pallidum einzusetzen.

Besondere Schwierigkeiten bereitete auch das Gedicht Nr. 7 („Schwindelhaber“; Lolium), dessen vier Strophen durch die zwei Synonyme zu Lolch enthaltende Refrainzeile "Schwindelhaber, Dippelhaber" geschlossen werden und in dem verschiedene wenig bekannte Ausdrücke für Getreidekrankheiten und Unkraut erscheinen, die metaphorisch für die falschen und kranken politischen Vorstellungen stehen, von denen die Köpfe gereinigt werden sollten. Der Vergleich war vorbereitet durch das Gleichnis vom Weizen und Unkraut im Evangelium des Matthaeus 13, 24-30, wo das dortige von „dem Feind“ gesäte Unkraut (genannt griechisch

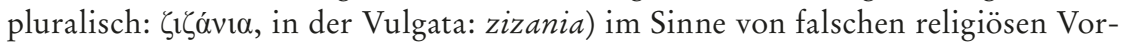
stellungen gedeutet wurde ${ }^{24}$. Uhlands erste Strophe lautet:

dern hier ein mühelos lesendes Verstehen in dem erforderlichen Sinn. Allerdings ist bereits Uhlands „wie er will“ elliptisch.

${ }^{23} \mathrm{Vgl}$. Belege für a consiliis seit Erasmus bei René Hoven, Lexique de la prose Latine de la Renaissance, Leiden 1994, S. 81.

${ }^{24} \mathrm{Vgl}$. zur theologischen Interpretation des Gleichnisses und der Kontroverse bei seiner Auslegung hinsichtlich der Behandlung von Häretikern Walther Ludwig, Supplementa Neolatina. Ausgewählte Aufsätze 2003-2008, ebd. hg. von Astrid Steiner-Weber, Hildesheim u. a. 2008 (Noctes Neolatinae 10), S. 272-275. 
Ei, wer hat in diesem Jahre

All den Wust ins Korn gebracht.

Mutterkorn und andere Waare,

Die im Kopfe dämisch macht,

Raden, Ruß, am meisten aber

Schwindelhaber, Dippelhaber?

Schwab beschaffte sich das einschlägige Unkraut- und Getreidekrankheitsvokabular aus Plinius, Nat. hist. 18, 44, 153-155, wo dieser lolium et tribulos („Lolch und Burzeldorn“) nennt und von robigo („Mehltau“, „Rost“, obsolet „Ruß“) spricht; auch orobanche („Sommerwurz“), aera und festuca (Synonyme zu lolium, obsolet „Raden“) werden dort von ihm erwähnt ${ }^{25}$. Einige dieser Bezeichnungen hatte schon Vergil in seine Georgica 1, 150-154 aufgenommen: ut mala culmos| esset robigo ... lappaeque tribolique interque nitentia cultal infelix lolium et steriles dominantur avenae („daß der böse Mehltau die Halme frißt ... Kletten und Burzeldorn [sc. kommen auf], und zwischen glänzenden Anpflanzungen breiten sich der unselige Lolch und der unfruchtbare Hafer aus"). Schwindelhaber und Dippelhaber waren heute obsolete volktümliche Bezeichnungen für den auch Taumellolch genannten Lolch, dessen Name aus dem lateinischen lolium entwickelt worden war und der angeblich Schwindelgefühle erregte und den Kopf benebelte („dämisch“ machte $)^{26}$. Schwab verwendete zudem - aus dem Gleichnis bei Matthaeus und damit direkt daran erinnernd - das griechische Fremdwort zizanium, das auch als Synonym zu lolium galt, und schrieb:

Quae Maga quisquilias frumento miscuit horno,

Aeras, heu, tribulos, orobanchen,

Festucasque soporiferas, tum, summa malorum,

Zizanium, loliumque malignum?

Etwas horazisches Kolorit fügen die Ausdrücke Maga und frumento ... borno hinzu (vgl. C. I 27, 21-22 Quae saga, quis te solvere Thessalis| Magus venenis, quis poterit deus? und C. 3, 23, 3-4 hornalfruge).

Als Uhland die Gedichte Nr.1-13 schrieb, war der Kampf um eine dem „alten guten Recht“ entsprechende neue Verfassung Gegenwart, und die zunächst einzeln publizierten Gedichte dienten diesem Kampf. Als Schwab sie in die horazische

25 Vgl. Gottfried Grosse, Caius Plinius Secundus Naturgeschichte übersetzt, Bd.5., Frankfurt am Main 1785, S. 214-218 (mit botanischer Kommentierung).

${ }^{26} \mathrm{Vgl}$. zu lolium temulentum, dem „Taumellolch“ K. G. Lutz, J. Sturms Flora von Deutschland in Abbildungen nach der Natur. Zweite, umgearbeitete Auflage, 3. Bd.: Echte Gräser, Gramineae, Stuttgart 1900, S.155-156. „Als Unkraut im Getreide, namentlich im Hafer, nicht selten. Die Körner sollen [...] giftig sein; die Fälle von Vergiftung durch unreines Mehl sind aber wohl richtiger auf die Beimengung von Mutterkorn zurückzuführen.“ 
Versform transformierte, dienten sie der bleibenden Erinnerung an die überstandene Zeit der Auseinandersetzungen. Umso wichtiger war dann das letzte Gedicht Nr. 14, das auf das mit König Wilhelm I. erreichte einigende Verfassungswerk zurückblickt und diesen König dafür rühmt (allerdings auch wieder ohne ihn namentlich zu nennen). Schwab ließ den letzten Vers - optisch damit über Uhland hinausgehend - in betonende Majuskeln setzen. Die 52 jambischen Trimeter dieses Gedichts enden mit den Worten (V. 46-52):

Sic est! in hisce temporum turbis feris

Suapte mente concitus princeps adest,

Dextramque genti ex mente grandi porrigit,

Pacturus ordinem atque jura libere.

Vidistis omnes, vosque testes advoco,

Suisque Clio mandet inde annalibus!

TV MACTE PRINCEPS, MACTA GENS ESTO MIHI!

Ja! mitten in der wildverworrenen Zeit

Ersteht ein Fürst, vom eignen Geist bewegt,

Und reicht hochherzig seinem Volk die Hand

Zum freien Bund der Ordnung und des Rechts.

Ihr habt's gesehen, Zeugen seyd ihr alle,

In ihre Tafeln grab' es die Geschichte!

Heil diesem König, diesem Volke Heil!

Zu Schwabs Unterrichtsaufgaben am Oberen Gymnasium in Stuttgart gehörte die Interpretation des Horaz ${ }^{27}$. Karl Klüpfel (1810-1894), einer seiner früheren Schüler an dieser Schule ${ }^{28}$ schrieb darüber 1858 in seiner Schwab-Biographie: „Mit besonderem Erfolg und Beifall erklärte er den Horaz, den er mit dichterischem Geiste auffaßte, und jeder empfängliche Schüler jener Zeit wird diese Stunden als Glanzpunkt des Unterrichts im Oberen Gymnasium in Erinnerung haben "29. Wo aber hatte Schwab selbst seine Lateinkenntnisse erworben und Horaz so sehr schätzen gelernt?

27 KLÜPfel, Gustav Schwab (wie Anm. 1) S. 94: „Die Fächer, die ihm [sc. als Professor am Oberen Gymnasium] übertragen wurden, waren: Interpretation des Horaz, griechische Anthologie, philosophische Schriften Ciceros, lateinische und griechische Stilübungen und römische Altertümer." Es wurde damals eine sehr reichliche Auswahl aus Horaz’ Oden, Epoden, Satiren und Episteln gelesen; zu den Stilübungen gehörte auch das Verfertigen lateinischer Verse.

${ }^{28}$ Vgl. Eugen Schneider, Art. „Klüpfel, Karl“, in: ADB, Bd.51, Leipzig 1906, S.244245. Klüpfel besuchte das Obere Gymnasium in Stuttgart, bevor er seit 1828 in Tübingen studierte, hatte also Schwab als Lehrer; 1841 heiratete er Schwabs Tochter Sophie.

29 Klüpfel, Gustav Schwab (wie Anm. 1) S. 94. 
Sein Vater, Johann Christoph Schwab (1743-1821), der 1778-1794 Professor für Philosophie an der Hohen Karlsschule in Stuttgart war ${ }^{30}$, achtete sehr auf die Erziehung seines jüngsten Sohnes. Er sorgte früh für seine schulische Unterrichtung $^{31}$. Gustav Schwab, „voll Eifer und Beharrlichkeit im Erlernen der Alten Sprachen" ${ }^{\text {"32 }}$, besuchte das Gymnasium in Stuttgart von der unteren bis zur oberen Abteilung, bis er 1809-1814 in Tübingen studierte, wo er sich der Studienordnung des Stifts gemäß zwei Jahre der Philologie und Philosophie und anschließend drei Jahre der Theologie widmete. Klüpfel zufolge waren es am Stuttgarter Gymnasium „Zwei Lehrer, die, beide in ihrer Art besonders tüchtig, eine nachhaltige Anregung auf Schwab ausübten. Der eine war Friedrich Roth, an der mittleren Abteilung der Anstalt tätig, der seine Schüler zu Fleiß zu nöthigen und in der Grammatik sattelfest zu machen wußte ${ }^{33}$.... Der andere war Drück, Professor am Oberen Gymnasium, ein liebenswürdiger humaner Mann, der in seiner ganzen Persönlichkeit die Klassische Bildung ausprägte und als Lehrer in hohem Grade geeignet war, in den Geist des Alterthums einzuführen“34. Und zu Schwabs philologischen Universitätsstudien vermerkt Klüpfel: „Am meisten scheint ihn der Philolog Conz angeregt zu haben, der mit der classischen Literatur wohl vertraut, ästhetisch gebildet war, als Dichter sich einen Namen gemacht und einige gelungene Übersetzungen von einzelnen Stücken der griechischen Dramatiker herausgegeben hatte. ... Er [sc. Schwab] sagte auch später öfters, daß er Conz manches verdanke ${ }^{\text {‘35. }}$.

\footnotetext{
30 Vgl. Wilhelm von Heyd, Art. „Schwab, Johann Christoph“, in: ADB, Bd. 33, Leipzig 1891, S. 157-158.

31 Klüpfel, Gustav Schwab (wie Anm.1) S.7: „Früh wurde Gustav in die Schule geschickt, und er erzählte oft, daß er sich genau erinnere, bald nach seinem vierten Jahr dort eine kurze lateinische Vorschrift, deren Worte er angab, abgeschrieben zu haben.“

32 Klüpfel, Gustav Schwab (wie Anm. 1) S. 12.

${ }^{33}$ Es handelt sich bei diesem Friedrich Roth um Christoph Friedrich Roth (1751-1813), vgl. zu ihm Karl Johann Friedrich (von) Rотн, Christophori Friderici Rothii A. M. Professoris Stuttgartiensis Laudatio, Stuttgart 1814. Er kam 1779 an das Stuttgarter Gymnasium und unterrichtete nach (vON) Roth, ebd., S.29, besonders die Autoren Cicero, Sallust und Livius. Alf Christophersen, Art. „Roth, Karl Johann Friedrich von“, in: NDB, Bd.22, Berlin 2005, S. 107, nennt als Todesjahr von Christoph Friedrich Roth fälschlich 1831 statt 1813. Für seine Tätigkeit vgl. auch Christoph Friedrich Rотн, Materialen zum Uibersetzen [sic] aus dem Teutschen in das Latein für die Jugend von 12-15 Jahren gesammelt und verfaßt, Stuttgart 1807. Das Buch enthält 402 deutsche, 1791-1806 zum Übersetzen gegebene Prosatexte mit lateinischen Ausdrücken als Übersetzungshinweisen.

34 Klüpfel, Gustav Schwab (wie Anm. 1) S. 14-15.

35 Klüpfel, Gustav Schwab (wie Anm. 1) S. 26-27.
} 
Friedrich Ferdinand Drück (1754-1807) ${ }^{36}$ und Carl Philipp Conz (1762-1827), der seit 1804 Professor für klassische Philologie an der Universität Tübingen war ${ }^{37}$, sind denn auch in verschiedener Weise für das Verhältnis von Schwab zu Horaz wichtig geworden. Drück war seit 1779 ein Kollege von Schwabs Vater an der Hohen Karlsschule gewesen und hatte dort die Alten Sprachen und Handelsgeographie unterrichtet, bis er nach der Schließung der Hohen Schule 1794 als Professor an das Obere Gymnasium in Stuttgart versetzt worden war. Er unterrichtete dort vor allem Latein, aber auch Griechisch ${ }^{38}$, Geschichte und Religion. Sein Schüler Karl Ludwig Roth (1790-1868) ${ }^{39}$ erklärte 1851: „Da er an den oberen Klassen lehrte, so hatte er weniger mit der Komposition, als mit der Erklärung der Autoren zu tun. ... Drück suchte unseren Geist vornehmlich dadurch zu bilden, daß er uns in den Geist der Autoren, zu meiner Zeit des Horaz und des Tacitus einführte. Nur er leitete uns an, die Gedanken des Klassikers aufzusuchen und zu erfassen ... “40. Und Conz schrieb in seinem Nekrolog auf ihn: „Unter den Römern waren Tacitus und Horaz seine Lieblingsschriftsteller ... [Er] verfaßte lateinische Gedichte ${ }^{\text {“41 }}$.

Conz gab 1810-1812 die kleineren Schriften Drücks in Tübingen in einer dreibändigen Sammelausgabe heraus. In dem mitveröffentlichten Verzeichnis der Subskribenten, das 252 Bestellungen aufführt, findet sich in Stuttgart „Schwab Oberjustizsekretair“ mit zwei Bestellungen, es ist der ältere Bruder von Gustav Schwab mit den Vornamen Karl Heinrich (1781-1847), der später geadelt wurde. Nicht

36 Vgl. Hartmann, Art. „Drück, Friedrich Ferdinand“, in: ADB, Bd.5, Leipzig 1877, S. 435. - Friedrich Ferdinand DRÜCK, Kleinere Schriften, 3 Bde., hg. von Carl Philipp Conz, Tübingen 1810-1812. - Karl Ludwig Rотн, Erinnerung an drei verdiente Gymnasiallehrer, Johann Andreas Werner, Christoph Friederich Roth, Friederich Ferdinand Drück. Eine Rede am Geburtstagsfest Seiner Majestät des Königs Wilhelm von Württemberg im Gymnasium in Stuttgart gehalten von dem Gymnasial-Rektor und Oberstudienrath Dr. Roth. Stuttgart 1851. - Herzog Karl Eugen von Württemberg und seine Zeit, hg. vom Württembergischen Geschichts- und Altertums-Verein, Bd. 2, Eßlingen 1909, S. 58 und 476.

37 Karl Klüpfel, Art. „Conz, Carl Philipp“, in: ADB, Bd. 4, Leipzig 1876, S. 457-458. Adalbert Elschenbroich, Art. „Conz, Carl Philipp“, in: NDB, Bd.3, Berlin 1957, S.347348.

${ }^{38}$ Drück erwarb offenbar auch neue gräzistische Fachliteratur. 1799 kaufte er ein Exemplar der griechisch-lateinischen, von dem Straßburger Professor Johann Schweighäuser mit einem sehr ausführlichem Kommentar herausgegebenen Ausgabe des Stoikers Epiktet (Epicteti Manuale et Cebetis Tabula, Leipzig 1798). Auf den Vorsatz schrieb er: „Drück 1799“. Dass der Schreiber dieses Eintrags Friedrich Ferdinand Drück war, bestätigt der nächste Besitzereintrag, der von 1807 stammt. Drücks Bibliothek kam also noch in seinem Todesjahr zum Verkauf. Das Exemplar befindet sich im Besitz des Verfassers.

39 Max Planck, Art. „Roth, Karl Ludwig“, in: ADB, Bd. 29, Leipzig 1889, S. 333-338. Er war einer der Söhne von Christoph Friedrich Roth, s. Anm. 33.

${ }^{40}$ Rотн, Erinnerung (wie Anm. 36) S. 12.

${ }^{41}$ Drück, Kleinere Schriften (wie Anm. 36) Bd. 3, S. XII. Conz leitete seine Schrift „Zum Andenken des am 27. April 1807 verstorbenen Professors am königl. obern Gymnasium und königl. Bibliothekars zu Stuttgart", ebenda S. VII-XXII, mit dem Zitat von Horaz ein (C. I 24, 6-8 cuipudor et iustitiae soror| incorrupta fides nudaque veritas, |quando ullum invenient parem?).

Zeitschrift für Württembergische Landesgeschichte 74 (2015), S. 121-148.

(C) Kommission für geschichtliche Landeskunde in Baden-Württemberg und Württembergischer Geschichts- und Altertumsverein e.V.

ISSN 0044-3786 
unter den in den Bänden 1-3 aufgeführten Subskribenten findet sich Gustav Schwab. Jedoch trägt das von der Bayerischen Staatsbibliothek München für das Internet digitalisierte Exemplar (Signatur: Philol. 93e-1) auf dem Vorsatz des ersten Bandes die Besitzersignatur „Gustav Schwab 1810“ und von seiner Hand auch den Eintrag „Subskr. Preis für beyde Bände 2 f. 24 kr.“ Spätestens jetzt lernte Schwab Drücks Carmina Latina kennen ${ }^{42}$.

$\mathrm{Zu}$ diesen gehört erstens die in 12 alkäischen Strophen verfaßte Ode Ad Franciam, die Drück schon 1794 in Tübingen in Band 3 der von Carl Viktor Hauff ${ }^{43}$ herausgegebenen Zeitschrift Philologie (Untertitel: „Eine Zeitschrift zur Beförderung des Geschmacks an griechischer und römischer Sprache und Literatur und eines gründlichen Studiums derselben") veröffentlicht, also in der Zeit geschrieben hatte, als Robespierre Schrecken verbreitete. Die Ode enthält einen leidenschaftlichen Appell an Frankreich, mit seinem Blutvergießen und seinen Kriegen aufzuhören. Die ersten drei Strophen lauten:

O quae vetustas mente nefaria

Pervertis oras, juraque gentium

Sacrasque leges, et cruento

Dilanias tua viscera ense;

Qualis, furorem cum traheret suo

Oetaeus heros pectore, dicitur

Insaniise, et dira Divis,

Dira sibi male adorsus ipsi:

Quando o! fugatam reddideris tibi

Terrisque pacem, Francia, gentibus

Exemplar olim gloriosum,

Nunc timor atque inhonesta cura,

„Oh, die du mit ruchlosem Sinn alte Länder und Völkerrechte und heilige Gesetze zerstörst und mit blutigem Schwert deine eigenen Eingeweide zerfleischst, wie der Held vom Oeta, als er in seinem Herzen raste, wahnsinnig gewesen sein und Schreckliches den Göttern, Schreckliches auch sich selbst übel angetan haben soll, oh wann wirst du, Frankreich, dir und den Ländern den von dir vertriebenen Frieden zurückgegeben haben, du, die du einst den Völkern ein ruhmrei-

42 Drück, Kleinere Schriften (wie Anm. 36) Bd. 3, S. 286-304.

43 Carl Viktor Hauff (1753-1832) war damals Professor am Seminar zu Maulbronn. Vgl. Zum Andenken an unseren seligen Vater M. Carl Viktor von Hauff, Dekan und Stadtpfarrer zu Cannstatt, Ritter des könig. Civil-Verdienst-Ordens, gestorben am 18. August 1832, Cannstatt 1832. 
ches Vorbild warst und die du jetzt Ursache von Furcht und schrecklicher Sorge bist, ..."

Die zerfleischten Eingeweide erinnerten den Leser an den Anfang von Lucans Bürgerkriegsepos, wo das ruhmreiche römische Volk seine siegreiche Rechte gegen seine eigenen Eingeweide richtet. Herkules, hier periphrastisch heros Oetaeus genannt, der im Wahnsinn seine eigene Frau und seine Kinder tötete, lässt an die Hercules furens betitelten Tragödien des Euripides und Seneca denken. In dem mythischen Vergleich und der pathetischen Anrede an die personifizierte Francia, deren Name erst in V. 10 erscheint (vgl. den entsprechenden Beginn mit $O$ und den auch spätgestellten Eigennamen des Adressaten in Horaz' C. II 7, 1-5), erkannten die Zeitgenossen Züge einer erfolgreichen Imitation des hohen Stils der politischen Lyrik des Horaz.

Drücks weitere sieben Gedichte sind alles Versübersetzungen. Die letzten drei sind deutsche und lateinische Übersetzungen von drei griechischen Epigrammen aus der Anthologia Graeca, wie sie seit dem 16. Jahrhundert oft hergestellt worden waren (hier nun aus der von Friedrich Jacobs [1764-1847] $]^{44}$ seit 1794 neu herausgegebenen Anthologia Palatina anstelle der altbekannten Anthologia Planudea). Die vier Gedichte zuvor sind lateinische Versübersetzungen von zeitgenössischen deutschen Gedichten. Zwei Gedichte von Karl Wilhelm Ramler (1725-1798), beginnend mit „Willst du den allerhöchsten Zeus erhöhen“ und mit „Klarer Bach, der hier unter Sträuchern“, wurden von Drück in alkäische Strophen bzw. in die Strophen des Asclepiadeum secundum (3 Asclepiadei + Glyconeus) übertragen, ein Gedicht von Christian Felix Weis(s)e (1726-1804), beginnend mit „Gütig hüllt in Finsternissen Gott die Zukunft ein“ erhielt sapphische Strophen, und ein Gedicht von Johann Peter Utz (1720-1796), beginnend mit „O Sonne, Königin der Welt“, erscheint in elegischen Distichen.

Danach scheint Drück Schwab nicht nur für Horaz gewonnen, sondern auch ein Vorbild für die Umsetzung zeitgenössischer Gedichte in horazische Strophenformen geboten zu haben. Der Rezensent in der Allgemeinen Literaturzeitung hat die Schwabsche Uhlandübersetzung mit anderen lateinischen Versübersetzungen zeitgenössischer deutscher Gedichte verglichen. Zuerst nannte er die Übersetzung des in deutschen Hexametern verfassten „Frühling“ von Ewald Christian von Kleist (1715-1759), die Georg Ludwig Spalding (1762-1811) noch als Student in lateinischen Hexametern 1783 in Berlin mit einer Vorrede seines Schwagers Friedrich Samuel Gottfried Sack (1738-1817) veröffentlicht hatte ${ }^{45}$. Sack hatte zur

${ }^{44}$ Vgl. zu ihm Manfred Fuhrmann, Friedrich Jacobs - Universalist der Klassischen Philologie, in: Wissenschaftsgeschichte zum Anfassen. Von Frommann bis Holzboog, hg. von Günther Bien u. a., Stuttgart-Bad Cannstatt 2002, S. 89-101.

${ }^{45}$ Georg Ludwig Spalding, Ver Poema Kleistii e Germanico latinum interprete G.L. Spalding, Praefatus est editor F.S.G. Sack, Berlin 1783. Vgl. Richard Hoche, Art. „Spalding, Georg Ludwig“, in: ADB, Bd.35, Leipzig 1893, S. 29-30. Spalding hatte bei Christian 
Rechtfertigung solcher Geistesspiele, die vielen unzeitgemäß erscheinen könnten, auf die Übersetzungen der deutschen Versepen des „Murner in der Hölle“ und des „Phaeton“ von Justus Friedrich Wilhelm Zachariä (1726-1777) in lateinische Hexameter durch Benedict Christian Avenarius (1771) ${ }^{46}$ und Heinrich Gottlieb Reichard (1780) ${ }^{47}$ hingewiesen.

Der Rezensent der Allgemeinen Literaturzeitung erinnerte außerdem an Gedichte von Klopstock und Schiller sowie an größere von Voß und Goethe, an denen sich „vor kurzem mehrere römische Verskünstler versucht“ hätten. Für Klopstock scheidet dadurch die Lessingsche Übersetzung von etwa 100 Versen aus dem 1. Gesang des Messias in lateinischen Hexametern, die er 1753 im 19. Brief des Zweiten Teils seiner Schriften veröffentlichte, ebenso aus wie die lateinische, auch in Hexametern gehaltene Übersetzung des 9. Gesanges von dem Piaristen Ludwig Bertrand Neumann (1726-1777), der 1770 in Wien Mors Christi seu Messias, Cantus IX ex illustri poemate Klopstockiano drucken ließ; und auch das Stück des Johann Baptist von Alxinger (1755-1797) aus dem 4. Gesang, das von Christoph Wilhelm Mitscherlich (1760-1854) in seine Eclogae recentiorum carminum latinorum (Hannover 1793) aufgenommen worden war, liegt für den Ausdruck „vor kurzem“ noch zu weit zurück. Unter diesen Umständen scheinen nur die drei Gedichte Klopstocks als von dem Rezensenten gemeint in Frage zu kommen, die der Professor Johann Dominicus Fuss (1781-1860) in Lüttich in seinen in Köln 1822 erschienenen Carmina Latina in lateinischer Form publizierte. Eines ist in elegischen Distichen, eines in asklepiadeischen und eines in alkäischen Strophen verfasst ${ }^{48}$.

Gottlob Heyne in Göttingen studiert. Das digitalisierte Exemplar der LUB Göttingen enthält die handschriftliche Widmung Heynio| Musarum Graecarum et Roma-|narum Flamini| $D$ [ono $][$ [edit $] D$ [edicavit $] \mid$ pii animi testificandi| causa| interpres.

46 Vgl. Sonja Martina Schreiner, Die komische Seite der Wissenschaftlichkeit: Avenarius' Aelurias, die neulateinische Übersetzung von Zachariäs Murner in der Hölle, in: Acta Conventus Neo-Latini Bonnensis: Proceedings of the Twelfth International Congress of Neo-Latin Studies (Bonn 2003), hg. von Rhoda Schnur u. a., Tempe/Arizona 2006, S. 747758, und Rosemarie Schillemeit (Hg.), Das Stammbuch des Benedict Christian Avenarius, Zeugnisse eines Studenten- und Hofmeisterlebens in Göttingen, Braunschweig und im Leipzig des jungen Goethe, Bielefeld 2002.

47 Sonja Martina Schreiner, Justus Friedrich Wilhelm Zachariä's „Der Phaeton“ and Heinrich Gottfried Reichard's Neo-Latin translation Phaethontis libri V, in: Humanistica Lovaniensia 53 (2004) S. 351-369, und Dies.: Phaet(h)on - mehrsprachig. Friedrich Wilhelm Zachariäs Der Phaeton (1754-1772) und Heinrich Gottfried Reichards Phaethontis libri quinque (1780), Frankfurt am Main u. a. 2005.

48 Johann Dominicus Fuss, Carmina Latina, additis e Germanico versis, in quibus Roma et Ars Graecorum A.W. Schlegel, et Ambulatio Fred. Schiller elegiae denuo emendatiores vulgatae, in caeteris Schilleri Campana et Goethei Alexis et Dora; praecedit de linguae cum universo ad scribendum tum ad poesin usu deque poesi et poetis neolatinis dissertatio, Köln 1822, S. 81-84, 98-100 und 121-122. Vgl. zu den Klopstock-Übersetzungen insgesamt EcHtermayer/Seyffert, Carmina (wie Anm. 16) S. IV-V. Zu Johann Dominicus Fuss vgl. jetzt Dieter Detiège, Zwei Gedichte von Josef Müller in Aachener Mundart und ins Neulateini-

Zeitschrift für Württembergische Landesgeschichte 74 (2015), S. 121-148.

(C) Kommission für geschichtliche Landeskunde in Baden-Württemberg und Württembergischer

Geschichts- und Altertumsverein e.V.

ISSN 0044-3786 
Lateinische Versübersetzungen von Schiller, Voß und Goethe, auf die sich der Rezensent bezieht, waren in der Tat aber monographisch kurz vor 1823 erschienen, und zwar waren sie alle in Stuttgart oder Tübingen gedruckt worden. Der Rezensent erwähnt ausdrücklich die Übersetzung der „Schiller'schen Glocke von Niethammer“. Hier handelt es sich um Jakob Benjamin Niethammer (1775-1847), der nach einem Studium im Tübinger Stift Pfarrer in Oppenweiler an der Murr war (1803-1825) und von dort aus diese Schrift veröffentlichte: Schillers Lied von der Glocke, und Schubarts Ode die Fürstengruft, in lateinische Verse übersetzt von J.B. Niethammer, Pfarrer in Oppenweiler, Tübingen: gedruckt bei Hopfer de l'Orme und zu haben bei dem Verfasser 1822. Er hatte die beiden Gedichte in rythmische und reimende Verse übersetzt, deren Stil durch ein Zitat der bekannten ersten Strophe der Glocke und der ersten Strophe aus Schubarts früher auch berühmtem, 1786 erstmals gedrucktem Gedicht deutlich werden soll (S. 5 und 39):

Fest gemauert in der Erden

Steht die Form, aus Lehm gebrannt,

Heute muß die Glocke werden,

Frisch Gesellen! seyd zur Hand.

Von der Stirne heiß

Rinnen muß der Schweiß,

Soll das Werk den Meister loben,

Doch der Segen kommt von oben.

Humo solido inhaeret

Forma luto coctili.

Hodie Campana sonet,

Promti este socii!

Opus caleat!

Sudor defluat!

Artifex ut celebretur,

At successus Dis debetur.

Da liegen sie, die stolzen Fürstentrümmer,

Ehmals Götzen ihrer Welt!

Da liegen sie, vom fürchterlichen Schimmer

Des blassen Tags erhellt!

Hic isti, quondam pie adorati,

Nunc cineres exanimes!

sche übertragen von Johann Dominik Fuß, in: Pro Lingua Latina 15, hg. von Hermann KrüsSel, Aachen 2014, S. 73-86. 
Hic isti inclyti sub umbris strati

Horrendis principes.

Niethammer ist also das Kunststück gelungen, die Reimform der deutschen Gedichte genau nachzuahmen. Bei der Wiedergabe der einzelnen Wörter war er dann notgedrungen zu Kompromissen gezwungen. Getroffen ist der Gesamtsinn, aber die Abweichungen im Wortlaut sind mitunter doch erheblich. Schwab entschied sich gegen eine solche mittelalterliche Form des Lateins, die der oben zitierte Rezensent „Mönchspoesie“ nannte, für die stilistisch als höherwertig geltende klassische horazische Odenform.

Bei den Wiedergaben längerer Vossischer und Goethischer Gedichte dachte der Rezensent an zwei Drucke von Benjamin Gottlob Fischer (1769-1846), der gleichfalls das Tübinger Stift durchlaufen hatte und 1810-1822 Professor an der Klosterschule bzw. dem Seminar in Schönthal war. Von dort veröffentlichte er: Loisa. Idyllion tribus eclogis absolutum auctore Johanne Henrico Voss. Latine vertit M. Benjamin Gottlob Fischer, Professor Seminarii Schoenthaliensis. Stuttgardiae, Sumtu Johannis Benedicti Metzleri, MDCCCXX. Und: Arminius et Theodora auctore Goethe. Latine vertit M. Benjamin Gottlob Fischer, Professor Seminarii Schoenthaliensis. Stuttgardiae, Sumtu Johannis Benedicti Metzleri, MDCC$\mathrm{CXXII}^{49}$. Beide Drucke haben auch eine deutsche Titelseite und enthalten jeweils auf einander gegenüberliegenden Seiten die lateinischen Hexameter Fischers und die deutschen Hexameter von Voß oder Goethe. Die jeweils ersten fünf Verse aus der „Luise“ und von „Hermann und Dorothea“ können einen Eindruck von der Qualität der Übersetzung geben:

Draußen in luftiger Kühle der zwo breitlaubigen Linden,

Die, von gelblicher Blüte verschönt, voll Bienengesurres,

Schattend der Mittagsstub', hinsäuselten über das Moosdach,

Hielt der redliche Pfarrer von Grünau heiter ein Gastmahl,

Seiner Luise zur Lust, hausväterlich prangend im Schlafrock.

Ecce foris, geminae latis ubi frondibus aestum

Defendunt tiliae, subflavo flore venustae,

Aedibus apricis tegmen, plenaeque susurris

Mellifluae pecudis, muscorum lene strepentes

Eminuere supra tectum - convivia dignus

Gronaviae pastor, tunica sublimis herili,

Dilectae laetus celebrabat festa Loisae.

49 Der Druck der Loisa hat am Anfang ein „Verzeichniß der Herren Pränumeranten“, das mit Ortsangabe durchweg Württemberger aufführt, die insgesamt 165 Exemplare bestellt haben. In Arminius et Theodora befindet sich das analoge Verzeichnis am Ende des Drucks. Es führt wieder ausschließlich Württemberger mit jetzt 199 bestellten Exemplaren auf. 
Hab ich den Markt und die Straßen doch nie so einsam gesehen! Ist doch die Stadt wie gekehrt! wie ausgestorben! Nicht fünfzig, Deucht mir blieben zurück, von allen unsern Bewohnern.

Was die Neugier nicht thut! So rennt und läuft nun ein jeder, Um den traurigen Zug der verarmten Vertriebnen zu sehen.

Tam solas numquam plateasque forumque videbam!

Urbs, purgata velut scopis, emortua tanquam

Cernitur! E cunctis mihi quinquaginta videntur

Vix superesse viris! Nova quantum cura videndi,

Hem, valet! Extorres ut cernat in agmine tristi

Unusquisque ruit curritque....

Fischer hat sich in seinen hübsch gelungenen Hexametern eng an den deutschen Wortlaut angeschlossen und so gut wie jede Bedeutungsnuance bewahrt und zugleich geklärt, dafür aber Raumüberschreitungen in Kauf genommen. Für die kurzversigen lyrischen Gedichte Uhlands kam eine solche Übersetzung in epische Hexameter natürlich nicht in Frage. Der Blick auf die Schwab damals vermutlich bekannten poetischen Übersetzungen zeigt, dass er einerseits für eine lateinische Übersetzung deutscher Gedichte nicht wenige Beispiele sah - diese Praxis war gut eingeführt und sollte noch weiter anwachsen - , andererseits für eine Übertragung der Uhlandschen Gedichte in horazische Odenmaße tatsächlich in erster Linie nur Drücks Gedichte zum Vorbild nehmen konnte. Bei seinem früheren Lehrer Drück fand er allerdings nur drei Beispiele für horazische Bearbeitungen zeitgenössischer Dichter, und zwar in der alkäischen, asklepiadeischen und sapphischen Strophenform.

Ob Schwab auch schon die horazisierenden Oden von Fuss in dessen Carmina Latina von 1822 kannte, der in ihnen außer Klopstock auch Gedichte von Voß, Hölty, Kleist, Salis und Utz bearbeitet hatte, ist ungewiss. Er könnte sie beachtet haben, aber da er sie erst kurz vor der Publikation seiner Gedichte kennen lernte, konnte er sie jedenfalls erst lesen, als er schon lange ein Freund und Kenner des Horaz geworden war. Er entschloss sich zu etwas, was noch nicht dagewesen war. Eine Sammlung von 14 deutschen Gedichten eines einzigen Verfassers hatte noch niemand im Lateinischen in ein breites Spektrum der horazischen Oden- und Epodenformen umgesetzt.

Die lateinische Versübersetzung des Gedichts von Voß durch Fischer 1820, von Goethe durch den gleichen Autor 1822, von Schiller und Schubart durch Niethammer 1822 und der Gedichte von Uhland durch Schwab 1823 - das ist eine Ballung lateinischer Versifikationen deutscher Gedichte, die in anderen deutschen Ländern zu dieser Zeit und in Württemberg im 19. Jahrhundert zu anderer Zeit nicht leicht zu finden ist. Die Autoren waren sich durchaus bewusst, dass die Öffentlichkeit lateinisches Dichten im allgemeinen nicht mehr für zeitgemäß hielt. Fischer schrieb 
1820 in seiner Praefatio: si versificationem latinam, inter obsoleta nostro tempore babitam, defendere aut quid exinde nos ipsi voluptatis aut discipuli nostri lucri ceperimus exponere instituamus, non tam praefationem quam librum scripsisse videamur („wenn wir uns vornähmen, das lateinische Dichten, das in unserer Zeit zu den abgetanen Dingen zählt, zu verteidigen oder zu erklären, was für eine Freude wir selbst und was für einen Gewinn unsere Schüler daraus erlangt haben, würden wir nicht so sehr ein Vorwort als ein Buch geschrieben zu haben scheinen“ ${ }^{50}$. Die Häufung der lateinischen Versübersetzungen in den Jahren 1820-1823 setzt nicht nur die vorzüglichen, auch aktiv verwendeten Lateinkenntnisse voraus, die Schüler von württembergischen Gymnasien und Klosterschulen bzw. Seminaren zu Beginn des 19. Jahrhunderts gewinnen konnten, sondern auch ein den Autoren gemeinsames Interesse, solche Kenntnisse in publizierte lateinische Versdichtungen umzusetzen.

Hierbei mag auch ein Buch eine Rolle gespielt haben, das hier noch nicht genannt und bisher auch nicht näher untersucht wurde. Es ist eine Anthologie vieler verschiedenartiger Beispiele neuzeitlicher lateinischer Dichtungen aus dem europäischen späten 15. bis späten 18. Jahrhundert, die August (Friedrich) Pauly (1796$1845)^{51}$, ein jüngerer Bruder des mit Gustav Schwab befreundeten, früh verstorbenen Ludwig August Pauly (1793-1812) ${ }^{52}$, unmittelbar nach Abschluss seines Studiums in Tübingen 1818 unter dem Titel Anthologia poematum Latinorum recentioris aevi veröffentlicht hatte ${ }^{53}$. Er widmete das Buch seinem gleichnamigen Vater (1756-1818), der ihn als Professor an der Klosterschule bzw. dem Seminar in Maul-

50 Bereits Sack hatte in der Vorrede zu Spaldings Versübersetzung des Gedichts von Kleist geäußert (SPAlding (wie Anm. 45) S. 10): Non me fugit profecto, nostro tempore et operam et oleum perdidisse vulgo credi illum, qui Latinis carminibus condendis manum admoverit („Es entgeht mir in der Tat nicht, daß in unserer Zeit, von dem, der es unternommen hat, lateinische Gedichte zu verfassen, gemeinhin geglaubt wird, daß er Mühe und Kosten verschwendet habe"; zu dem sprichwörtlichen operam et oleum perdere vgl. Erasmus, Adag. 1.4.62).

51 Vgl. Wilhelm Sigmund Teuffel, Art. „Pauly, August von“, in: ADB, Bd.25, Leipzig 1887, S. 297-298. - Walter Hagen, August von Pauly, Archäologe und Philologe, in: Schwäbische Lebensbilder, Bd. 6, Stuttgart 1957, S. 205-210. - Monika Balzert, Art. „Pauly, Gottlieb Wilhelm August (auch August Friedrich) von“, in: NDB, Bd. 20, Berlin 2001, S. 136-137. Bekannter als durch seine neulateinische Anthologie ist dieser August Pauly, der seit 1831 Professor am Oberen Gymnasium in Stuttgart war, durch seine seit 1839 veröffentlichte und nach seinem Tod 1864 in 6 Bänden zum Abschluss gebrachte „Real-Encyclopädie der classischen Alterthumswissenschaft" geworden, da diese in ihrer 1893-1980 herausgegebenen, 84 Bände umfassenden Neubearbeitung den Titel „Paulys Real-Encyclopädie der classischen Altertumswissenschaft“ trägt und Paulys Name sogar in den sogenannten, 1964-1975 publizierten „Kleine(n) Pauly“ und den seit 1996 erscheinenden „Neue(n) Pauly“ übernommen wurde.

52 Vgl. Krauss, Schwäbische Literaturgeschichte (wie Anm. 17) S. 22-23.

53 August Friedrich Pauly (Hg.), Anthologia poematum Latinorum aevi recentioris curavit Augustus Pauly A.L.M. [= Artium Liberalium Magister] Tubingae Apud Henricum Laupp, MDCCCXVIII. 
bronn unterrichtet und auch später in seinen Studien wohl angeregt hatte ${ }^{54}$, kurz vor dessen Tod und außerdem seinem Freund Friedrich Heinrich Kern (1790$1842)^{55}$, der sein Repetent im Tübinger Stift gewesen und nun Professor an der Klosterschule bzw. dem Seminar in Blaubeuren war. Pauly schreibt im Vorwort, dass er nach seinem Studium der antiken Autoren auch die neuzeitlichen lateinischen kennen lernen wollte und dabei qualitativ sehr Unterschiedliches gefunden habe. Tamen, cum inter quisquilias meliora hand raro delitescere, quae una cum caeteris respuere fastidiosius foret, recte mibi persuasissem, depromere ex copiosa illa farragine invenibusque praesertim amicis proponere aliqua, unde oblectationem forsan et fructum capere possent, consilium cepi. („Dennoch habe ich, als ich mich mit Recht überzeugt hatte, daß unter dem Wust nicht selten Besseres verborgen war, das mit dem übrigen zu verschmähen allzu anmaßend wäre, den Plan ge$\mathrm{fa} \mathrm{Rt}$, aus diesem reichlichen Mischfutter etwas hervorzuholen und besonders meinen jungen Freunden vorzusetzen, woraus sie vielleicht Vergnügen und Nutzen holen konnten"; farrago hatte für geistige Kost zuerst Juvenal verwendet).

Er wählte insgesamt 62 Autoren (davon bleiben 2 anonym) und 268 Dichtungen aus den ihm in Tübingen verfügbaren Büchern aus (er erklärt, manche, die er lesen wollte, nicht vorgefunden zu haben), ordnete die Texte in drei nach Literaturgattungen und metrischen Formen gesonderte Abteilungen und einen Anhang, und bot 12 Heroica, also hexametrische Gedichte, 74 Lyrica, also Gedichte, die in der Regel in horazischen Oden- und Epodenformen abgefasst sind, 181 Elegica, Epigrammata, Gnomica, Aenigmata, die in der Regel in elegischen Distichen (Hexameter + Pentameter) komponiert sind, und als Appendix ein Drama, dessen Sprechverse in jambischen Trimetern erscheinen. Damit waren alle häufigeren lateinischen Versmaße repräsentiert. Innerhalb der Abteilungen suchte er die Gedichte prinzipiell chronologisch vom Ende des 15. bis zum Ende des 18. Jahrhunderts zu ordnen $^{56}$. Dem Textteil folgen am Ende lateinische Kurzbiographien der Autoren mit Quellenangaben.

Die Anthologie gibt auf 316 Seiten natürlich nur eine äußerst knappe Auswahl, in der jeder Kenner viele Autoren vermisst. Einerseits ist es aber erstaunlich, wie viele neulateinische Dichter der 22-Jährige schon gelesen hatte, andererseits konnte

${ }^{54}$ Der Vater Pauly zählte auch zu den Subskribenten der Ausgabe der Kleineren Schriften von Friedrich Ferdinand DrüCK (wie Anm. 36). Für die Vermutung von Balzert, Pauly (wie Anm. 51): „Eine 1818 in Tübingen erschienene Anthologie neulateinischer Dichtung stammt wohl vom Vater" scheint es mir aber keine ausreichenden Anhaltspunkte zu geben.

${ }^{55}$ Vgl. Heinrich Julius Holtzmann, Art. „Kern, Friedrich Heinrich“, in: ADB, Bd. 15, Leipzig 1882, S. 632.

${ }^{56}$ Darunter befinden sich auch Erstveröffentlichungen von Gedichten der Württemberger Gottfried Ulrich David Kapff (1768-1815, Superintendent Herrenberg, 1812-1815 Dekan in Wildbad) und Karl Philipp Friedrich Kurrer (1749-1827, Superintendent Reutlingen). In: Die Handschriften der Württembergischen Landesbibliothek Stuttgart, bearbeitet von Ingeborg Krekler u.a., Bd. 1, Wiesbaden 1963, S. 213, wird Cod. poet. et phil. $8^{\circ} 35$ mit Carmina Latina von Karl Philipp Friedrich Kurrer beschrieben. 
wohl nur ein 22-Jähriger ein solch immenses Vorhaben überhaupt wagen. Anthologien hatte es in der neuzeitlichen lateinischen Literatur schon manche gegeben. In den Bänden seiner Deliciae poetarum Belgicorum, Gallorum, Germanorum und Italorum hatte Janus Gruter zu Anfang des 17. Jahrhunderts die neuzeitliche lateinische Dichtung mehrerer Nationen gesammelt und für mehrere andere Nationen Nachfolger gefunden. Meist enthielten die Anthologien eine Auswahl zeitgenössischer Dichtungen, so die Carmina poetarum nobilium von Joannes Paulus Ubaldinus (Mailand 1563) und zuletzt auch die Eclogae recentiorum carminum latinorum des Heyne-Schülers Christoph Wilhelm Mitscherlich ${ }^{57}$ von 1793, zu denen Christian Gottlieb Heyne (1729-1812) selbst Gedichte beigesteuert hatte und die neben Gedichten von Mitscherlich auch solche von Avenarius, Denis und Hoeuft enthielten ${ }^{58}$. Den Versuch, in einem einzigen Band eine Auswahl von lateinischen Gedichten aller Gattungen aus drei Jahrhunderten bis kurz vor die eigene Zeit und aus vielen Ländern Europas zu bieten, hatte vor Pauly in Deutschland noch niemand gewagt.

Das einzige zuvor erschienene und etwas vergleichbare Werk von Jean Brunel, Le Parnasse Latin moderne ou choix des meilleurs morceaux des poètes latins qui se sont le plus distingués depuis la renaissance des lettres jusqu'à nos jours, avec leurs notices et la traduction françoise; à l'usage des Professeurs de latinité, Lyon 1808, kannte Pauly nicht (es ist noch heute weder in Tübinger noch in Stuttgarter öffentlichen Bibliotheken zu finden), und es ist auch ganz anders als sein eigenes Werk angelegt. Brunel möchte, wie er im Vorwort schreibt, die jungen Leser von Autoren wie Horaz, Vergil und Ovid mit Autoren wie Rapin, Vida und Commire erfreuen und hat dazu neulateinische Gedichte in zwei umfangreichen Bänden von je fast 500 Seiten gesammelt. Die insgesamt sogar 110, allerdings oft nur mit wenigen Versen exzerpierten Autoren folgen einander mit ihren Kurzbiographien in alphabetischer Reihe von Accolti bis Ménage im ersten, von Métivier bis Wallius im zweiten Band, wohinter noch eine kleinere Anzahl von Autoren ohne Kurzbiographie von Albani bis B** d'Arles steht. Das ist das einzige Ordnungsprinzip. Die Autoren sind fast alle Franzosen oder Italiener. Nur sieben Niederländer finden sich (Barlaeus, Petrus Burmanus, Grotius, Nicolaus Heinsius, Sidronius Hoschius S.J., Johannes Secundus, Wallius S.J.), zwei Briten (Buchanan, Owen) und ein Pole (Sarbievius). An Deutschen entdeckt man in dem biographielosen Anhang schließlich Bidermann, Lauterbach und Melissus (letzteren nur mit zwei Distichen). Brunel kannte sich nur bei den Franzosen und Italienern gut aus und ließ den chronologischen und gattungsmäßigen Aspekt bei seiner Anordnung außer Acht.

Wie Pauly vorging und was er aufnahm, soll ein Blick auf die Abteilung der Lyrica (S.33-137) lehren. Die ersten drei Autoren stammen noch aus dem 15. Jahr-

57 Vgl. Franz Eyssenhardt, Art. „Mitscherlich, Christoph Wilhelm“, in: ADB, Bd.22, Leipzig 1885, S. 15.

58 Vgl. zu den neulateinischen Anthologien allgemein Josef IJsew IJN/Dirk SACRÉ, Companion to Neo-Latin Studies, Part II, Leuven 1998, S. 479-487. 
hundert: Die Liste beginnt mit einer sapphischen Ode des italienischen Humanisten Actius Sincerus Sannazarius (1458-1530) auf dessen Villa Mergillina, für die er auf die Deliciae Poetarum Italorum zurückgreifen musste, da er keine Ausgabe dieses Autors zur Verfügung hatte ${ }^{59}$. Es folgen eine alkäische Ode des deutschen Humanisten Eobanus Hessus (1488-1540) aus den Deliciae Poetarum Germanorum $^{60}$, und ein lyrisches Epitaphium von Dietrich von Bülow (1460-1523) ${ }^{61}$, einem Freund von Hessus, zitiert aus einem soeben erschienenen Werk, aus Gottlieb Christian Friedrich Mohnike, Ulrich Hutten's Klagen gegen Wedeg Loetz und dessen Sohn Henning, Greifswald 1816.

Ganz dem 16. Jahrhundert gehören vier Autoren an: Es gibt sechs Gedichte des Niederländers Johannes Secundus (1511-1536) aus einer Pariser Ausgabe von $1748^{62}$ und eines von dem Franzosen Marcus Antonius Muretus (1526-1555) nach der Ausgabe von Ruhnken (Leiden 1789) ${ }^{63}$, drei von dem Deutschen Petrus Lotichius Secundus (1528-1560) nach der Ausgabe von Kretschmar (Dresden 1773) ${ }^{64}$ und eines von dem Deutschen Michael Hasslobius (1540-1589) nach den Deliciae Poetarum Germanorum ${ }^{65}$.

Es folgen noch Gedichte von vier Autoren aus dem 17. Jahrhundert: Martin Opitz (1597-1639) erscheint mit zwei Gedichten nach einer Breslauer Ausgabe von $1625^{66}$, Hugo Grotius (1583-1645) ebenfalls mit zwei nach der Leidener Ausgabe von $1645^{67}$. Anschließend sind die Jesuiten Matthias Casimir Sarbievius (1578-1640) ${ }^{68}$

59 Nr. 13: Jacobus Sannazarius, Opera omnia Latine scripta, Venedig 1535, Bl. 31 (Epigr. 1, 2). Die Nachweise dienen der Identifizierung. Sie werden meist nicht aus den von Pauly benützten Ausgaben entnommen.

60 Nr. 14: Helius Eobanus Hessus, Operum farragines duae, Frankfurt 1564, S. 473-474 (Sylvae 4, 19).

${ }^{61}$ Nr. 15. Vgl. Heinrich Grimm, Art. „Bülow, Dietrich von“, in: NDB, Bd. 2, Berlin 1955, S. 733 .

${ }^{62}$ Nr. 16: Petrus Scriverius (Hg.), Joannis Secundi Opera, Leiden 1631, S. 130-131 (Od. 2), Nr.17: S.136-137 (Od. 9), Nr.18: S.137-138 (Od. 11), Nr. 19: S.107-109 (Epigr. 34), Nr. 20: S. 223-225 (Sylv. 3), Nr. 21: S. 225-227 (Sylv. 4).

${ }^{63}$ Nr. 22: Marcus Antonius Muretus, Iuvenilia, Paris 1553, S.123-124 (Od. 5 Bacchus poetas facit et fovet), in späteren Auflagen seiner Poemata: Carm. 2, 15.

${ }_{64}$ Nr. 23: Petrus Burmanus Secundus (Hg.), Petri Lotichii Secundi Poemata omnia, Amsterdam 1754, S. 435-438 (Carm. 1, 19), Nr. 24: S. 561-563 (Carm. 3, 32), Nr. 24b: S.451452 (Carm. 1, 30).

${ }_{65}$ Nr. 25: (Janus Gruterus), Deliciae poetarum Germanorum huius superiorisque aevi illustrium collectore A.F. G. G., Pars III, Frankfurt am Main 1612, S. 491-492.

${ }^{66}$ Nr. 26: Martin Opitz, Weltliche Poemata, Breslau 1690, S. 325 (Silv. 1), Nr. 27: S. 351 (Silv. 3).

67 Nr. 28: Hugo Grotius, Poemata, Leiden 1639, S.232-233 (Farrag. 2), Nr. 29: S.260261 (Farrag 3).

68 Nr. 30: Matthias Casimir Sarbievius, Opera poetica, Breslau 1753, S. 3-4 (Lyr. 1, 2), Nr. 31: S. 30-32 (Lyr. 1, 16), Nr. 32: S. 35-36 (Lyr. 1, 19), Nr. 33: S. 49-52 (Lyr. 2, 5), Nr. 34: S. 52-53 (Lyr. 2, 6), Nr. 35: S. 60-61 (Lyr. 2, 12), Nr. 36: S. 65-68 (Lyr. 2, 16), Nr. 37: S. 78-79 (Lyr. 2, 23), Nr. 38: S. 90-91 (Lyr. 3, 4), Nr. 39: S. 142-144 (Lyr. 4, 3), Nr. 40: S. 166-167 (Lyr. 4, 15), Nr. 41: S. 177 (Lyr. 4, 23), Nr. 42: S. 182-183 (Lyr. 4, 26), Nr. 43: S. 199-200 (Lyr. 4, 36). 
aus Polen und Jacob Balde aus Deutschland (1604-1668) ${ }^{69}$ mit 14 bzw. 30 Gedichten vertreten. Es sind überraschenderweise die Autoren, die mit Abstand am meisten Gedichte in den Lyrica haben. Die poetische Leistung von Sarbievius konnte in der frühen Neuzeit der des Horaz gleichgesetzt werden ${ }^{70}$; Balde war von Herder empfohlen und übersetzt worden ${ }^{71}$. Für beide Autoren lagen Pauly nach seinen Angaben mehrere Ausgaben vor.

Fünf ausschließlich deutsche Autoren aus dem 18. Jahrhundert, deren Ausgaben ihm zur Verfügung standen, sind mit wenigen Gedichten vertreten: Aus den Varii generis Carmina Latina von Georg Friedrich Müller nahm Pauly ein Gedicht (Lisbonae excidium $)^{72}$, aus Christian Adolph Klotz (1738-1771), Carmina omnia, kamen sieben ${ }^{73}$, von Michael Denis (1729-1800), Carmina quaedam, zwei ${ }^{74}$, aus Mitscherlichs Eclogae von 1793 eines $^{75}$, und aus Drücks schon besprochenen Carmina Latina seine Ode Ad Franciam aus dem Jahr 1794 (Nr. 85).

Es würde hier zu weit führen, wenn man die Themen der Gedichte im einzelnen aufführen wollte. Die verschiedenen Autoren lassen mit Recht erwarten, dass viele poetische Themen angesprochen wurden. Für die Gedichte in den anderen Abteilungen seien hier nur die Namen der Autoren in der Reihenfolge ihres Vorkommens genannt, wobei Vornamen nur bei möglichen Missverständnissen hinzugefügt sind. Die meisten der im Folgenden Genannten gehören heute zu den in Fachkreisen bekannteren neulateinischen Autoren. Dies gilt insbesondere für die, die dem 15.-17. Jahrhundert angehören. In den Heroica erscheinen Vida, Rapinus, Sannazarius, Lotichius Secundus, Grotius, Klotzius, Henricus Godofredus Reich-

69 Nr. 44: Jacob Balde, Poemata, Tomus I, Köln 1660, S. 19-20 (Lyr. 1, 13), Nr. 45: S. 37 (Lyr. 1, 26), Nr. 46: S. 42-45 (Lyr. 1, 33), Nr. 47: S. 46-48 (Lyr. 1, 35), Nr. 48: S. 82-83 (Lyr. 2, 19), Nr. 49: S. 85-86 (Lyr. 2, 21), Nr. 50: S. 87-88 (Lyr. 2, 23) Nr. 51: S. 100-101 (Lyr. 2, 33), Nr. 52: S. 111 (Lyr. 2, 40), Nr. 53: S. 113-115 (Lyr. 2, 43), Nr. 54: S. 116-117 (Lyr. 2, 46), Nr. 55: S. 117-118 (Lyr. 2, 47), Nr. 56: S. 127 (Lyr. 3, 3), Nr. 57: S. 139-141 (Lyr. 3, 12), Nr. 58: S. 146147 (Lyr. 3, 17), Nr. 59: S. 154-155 (Lyr. 3, 25), Nr. 60: S. 180-181 (Lyr. 3, 43), Nr. 61: S. 189190 (Lyr. 3, 48), Nr. 62: S. 201-202 (Lyr. 4, 5), Nr. 63: S. 203-204 (Lyr. 4, 7) Nr. 64: S. 204-208 (Lyr. 4, 9), Nr. 65: S. 221-222 (Lyr. 4, 19), Nr. 66: S. 222-223 (Lyr. 4, 20), Nr. 67: S. 236-238 (Lyr. 4, 30), Nr. 68: S. 258-259 (Lyr. 4, 48), Nr. 69: S. 385-386 (Silv. 3, 6), Nr. 70: S. 392-395 (Silv. 4, Threnod. 1), Nr. 71: S. 602 (Silv. 9, 9), Nr. 72: S. 612-614 (Silv. 9, 14), Nr. 73: S. 50 (Lyr. $1,38)$.

70 Vgl. Ludwig, Opuscula (wie Anm. 6) S. 85-86.

71 Sie sind zusammengestellt in: Johann Gottfried von Herder, Sämmtliche Werke. Zur schönen Literatur und Kunst. Zwölfter Teil, Stuttgart/Tübingen 1829, S. 181-324.

72 Nr. 74: Georg Friedrich Müller, Varii generis Carmina Latina, denuo excusa cum Additamentis, Annaberg 1769, S. 33-36.

73 Nr.75: Christian Adolph Klotz, Carmina omnia, ohne Ort 1766, S.7-10 (Od. 3), Nr. 76: S. 13 (Od. 5), Nr. 77: S. 21-23 (Od. 9), Nr. 78: S. 50-53 (Od. 24), Nr. 79: S. 55-56 (Od. 26), Nr. 80: S. 57-58 (Od. 27), Nr. 81: S. 3-6 (Od. lib. 2).

74 Nr. 82: Michael Denis, Carmina quaedam, Wien 1794, S. 146, Nocti, Nr. 83: S. 147, Musis.

75 Nr. 84: Christoph Wilhelm Mitscherlich (Hg.), Eclogae recentiorum carminum Latinorum, Hannover 1793, S. 147-150 (Ad Petrum Leopoldum Imp. Aug.). 
hardus, Morcelli bei Mitscherlich und Hieronymus de Bosch, in den Elegica etc. Sannazarius, Naugerius, Molsa, Buchananus, Cordus, Johannes Fabricius, Fidler, Lauterbach, Lindenberg, Hutten, Melanchthon, Melissus, Micyllus, Posthius, Sabinus, Stigelius, Georgius Tilenus, Nicodemus Frischlinus, Dominicus Baudius, Josephus Scaliger, Daniel Heinsius, Johannes Secundus, Janus Vitalis, Joannes Jovianus Pontanus, Cunradinus Henning, Grotius, Passeratius, Julius Caesar Scaliger, Elias Cuchler, Josephus Langius, Martinus Opitius, Eobanus Hessus, Lotichius Secundus, Muretus, Caspar Barlaeus, Sidronius Hoschius S.J., Wallius S.J., Klotzius, Laurentius van Santen, Hoeuft, de Bosch, Spalding, Heyne, Vopiscus Horatius Acker, Friedrich Wilhelm Döring, Mitscherlich, Abraham Gotthelf Kaestner, Godofredus Ulricus David Kapf und Carolus Fridericus Philippus Kurrer. Das angehängte Drama Alexander trans Tanaim ist von dem Österreicher Michael Denis, der stofflich aus der Alexandergeschichte des Curtius Rufus schöpfte. Aus Deutschland stammen insgesamt 36 Autoren (die der Lyrica miteinbezogen), mit Abstand folgen 11 Niederländer und 8 Italiener. Spärlich sind die Franzosen mit nur drei vertreten, dazu kommen ein berühmter Schotte und ein berühmter Pole. Wie bei Brunel überwiegen die Landsleute, wenn auch proportional nicht so stark wie bei jenem.

Pauly konnte also trotz bemerkbarem Büchermangel, der für manche Lücken verantwortlich ist, ein gewisses, chronologisch und metrisch geordnetes Bild der lateinischen Dichtung von Schottland bis Italien und von Frankreich bis Polen mit einem deutsch-niederländischen Zentrum während der neuzeitlichen Jahrhunderte zusammenstellen, das dem Leser zahlreiche Autoren mit einer Vielfalt von Gedichtformen und Themen nahebrachte. Fast die Hälfte der Gedichte der Lyrica sind von Balde, was für eine entsprechende Schätzung des Dichters zeugt, zu der vermutlich Herders Empfehlung maßgeblich beigetragen hat. In seinem Vorwort erklärt Pauly zu Balde und Sarbievius: Baldaeum, Sarbievium flammis vere piis accensos sublimiori nonnunquam plectro cecinisse minime eo infitias („Ich leugne nicht im geringsten, daß Balde und Sarbievius von wahrhaft frommen Feuern entzündet mehrfach in höherem Stil gedichtet haben").

Wenn man die Autorenlisten überblickt, fällt auf, dass die beiden größten Abteilungen, die Lyrica und die Elegica, Epigrammata, Gnomica, Aenigmata mit Württembergern aus Paulys Lebenszeit enden. Es sind einerseits Friedrich Ferdinand Drück (1754-1807) mit seiner Ode von 1794 (Nr. 85) und andererseits Gottfried Ulrich David Kapff (1768-1815) und Karl Philipp Friedrich Kurrer (17491827) mit einigen bisher sogar ungedruckten Gedichten, einer Elegie über den Winter, Rätseln sowie scherzhaften und gnomischen Epigrammen (Nr. 241-266). Die Gedichte von Kapff und Kurrer sind die einzigen, die Pauly nicht gedruckten Ausgaben entnahm. Er, dessen Mutter eine geborene Kapff war, schreibt, Franz Kapff habe ihm die Gedichte seines Vaters zur Verfügung gestellt, und er dankt Kurrer, dass er ihm erlaubte, aus dessen Gedichten, was er wollte, auszuwählen: Quae inserui Viri doctissimi poeseosque Romanae peritissimi carmina nonnulla, 
debeo insigni ipsius erga me benevolentiae, quae ex uberrima poematum et ornatissima penu depromere mibi quaeque vellem, indulsit („Ich habe einige Gedichte des hochgelehrten und in der römischen Dichtung sehr kompetenten Mannes aufgenommen und schulde dies eben seinem besonderen Wohlwollen gegenüber mir, das mir erlaubte, aus dem überreichen und wunderschönen Vorrat seiner Gedichte alles herauszuholen, was ich wollte“). Er bedauere nur, dass er Kurrers längere, Pagus Langenbrandensis betitelte Dichtung über das Schwarzwalddorf Langenbrand ihres Umfangs von drei Büchern wegen nicht auch in seine Anthologie aufnehmen konnte.

Paulys Anthologie wird zunächst in Württemberg Verbreitung bei gebildeten Lesern gefunden haben ${ }^{76}$. Sie werden es auch geschätzt haben, zu sehen, wie die neuzeitliche Tradition des lateinischen Dichtens, die sie hier beispielhaft kennenlernen konnten, auch Württemberger um 1800 einschloss. Und einige wie Fischer, Niethammer und Schwab mochten sich dadurch auch bestärkt gefühlt haben, wenn sie diese Tradition ihrerseits fortsetzen wollten. Dass sie es in der Form von Versübersetzungen deutscher Dichtungen taten, ist freilich nicht nur ihrer individuellen Eigenart zuzuschreiben, sondern auch dem Umstand, dass, während freie lateinische Kompositionen in dieser Zeit wegen des Aufblühens der nationalsprachlichen Literatur abgenommen hatten, die Wiedergabe von zeitgenössischen deutschen Dichtungen in lateinischen Versen in der zweiten Hälfte des 18. Jahrhunderts bei entsprechend vorgebildeten Lesern im Ansehen gestiegen war.

\footnotetext{
76 Sie ist in Bibliotheken außerhalb Deutschlands selten geblieben. Ich benütze jedoch ein Exemplar, das das Schicksal hatte, zweimal den Atlantik zu überqueren. 1881 wurde es erworben von Edward Parmelee Morris (1853-1938), B. A. Yale University 1874, Professor of Latin Language and Literature Yale 1891-1919. Das Buch gelangte aus seinem Besitz an seinen Neffen Austin Morris Harmon (1878-1950), B. A. Williams College 1902, Ph. D. Yale 1908, Professor of Greek and Latin Yale 1916-1945 und aus dessen Besitz an seinen Schwiegersohn Howard Newton Porter (1917-1993), B. A. Yale 1938, Ph. D. Yale 1942, Professor of Greek and Latin Columbia University 1956-1978, der mir das Buch 1973 in New York schenkte.
} 